Draft version OCtober 30, 2018

Preprint typeset using $\mathrm{L}^{A} \mathrm{~T}_{\mathrm{E}} \mathrm{X}$ style emulateapj v. 11/10/09

\title{
ANALYTICAL STAR FORMATION RATE FROM GRAVOTURBULENT FRAGMENTATION
}

\author{
PATRICK HenNeBELle \\ Laboratoire de radioastronomie, UMR CNRS 8112, \\ École normale supérieure et Observatoire de Paris, \\ 24 rue Lhomond, 75231 Paris cedex 05, France \\ AND \\ Gilles Chabrier \\ École normale supérieure de Lyon, CRAL, UMR CNRS 5574, 69364 Lyon Cedex 07, France \\ School of Physics, University of Exeter, Exeter, UK EX4 4QL \\ (Dated:) \\ Draft version October 30, 2018
}

\begin{abstract}
We present an analytical determination of the star formation rate (SFR) in molecular clouds, based on a time-dependent extension of our analytical theory of the stellar initial mass function (IMF). The theory yields SFR's in good agreement with observations, suggesting that turbulence is the dominant, initial process responsible for star formation. In contrast to previous SFR theories, the present one does not invoke an ad-hoc density threshold for star formation; instead, the SFR continuously increases with gas density, naturally yielding two different characteristic regimes, thus two different slopes in the SFR vs gas density relationship, in agreement with observational determinations. Besides the complete SFR derivation, we also provide a simplified expression, which reproduces reasonably well the complete calculations and can easily be used for quick determinations of SFR's in cloud environments. A key property at the heart of both our complete and simplified theory is that the SFR involves a densitydependent dynamical time, characteristic of each collapsing (prestellar) overdense region in the cloud, instead of one single mean or critical freefall timescale. Unfortunately, the SFR also depends on some ill-determined parameters, such as the core-to-star mass conversion efficiency and the crossing timescale. Although we provide estimates for these parameters, their uncertainty hampers a precise quantitative determination of the SFR, within less than a factor of a few.
\end{abstract}

Subject headings: stars: formation — ISM: clouds — physical processes: turbulence

\section{INTRODUCTION}

The determination of the star formation rate (SFR) in molecular clouds and in galaxies is one of the main challenges of star formation theory. In the modern paradigm of star formation, stars form out of prestellar cores which result from the gravo-turbulent fragmentation of molecular clouds (e.g. MacLow \& Klessen 2004). Within the past few years, two analytical approaches have emerged, aiming at characterizing the SFR issued from the probability density function (PDF) of density fluctuations induced by turbulence in the cloud (Krumholz \& McKee 2005-KM, Padoan \& Nordlund 2011-PN). Both theories rely on (i) a density threshold, whose nature differs in each case, for star formation, (ii) one characteristic dynamical timescale, defined either at the cloud's mean density or at the threshold density. In this Letter, we derive a SFR, based on our IMF analytical theory (Hennebelle \& Chabrier 2008-HC08, 2009-HC09, Chabrier \& Hennebelle 2011), and show that (i) this theory yields SFR values in good agreement with observations, (ii) there is no a priori density threshold for star formation; instead, the SFR continuously increases with gas density, with indeed two different regimes. We also show that the exact value of the SFR depends on the combination of some ill-determined parameters, notably the core-to-star efficiency and the crossing timescale, whose uncertainties, and dependence upon cloud conditions, hamper an exact determination of the SFR.

\section{STAR FORMATION RATE: THEORIES}

We first summarize the previous SFR theories by KM and PN. We then briefly present the SFR derived from a time-dependent extension of our theory of star formation, which will be presented in details in a forthcoming paper. Finally we present a simplified version of this theory which, alternatively, can be seen as an improved KM or PN theory.

Following Krumholz \& McKee (2005), we define the dimensionless star formation rate per free-fall time, $S F R_{f f}$, as the fraction of cloud mass converted into stars per cloud mean free-fall time, $\tau_{f f}^{0}$, i.e.: $S F R_{f f}=\frac{\dot{M}_{*}}{M_{c}} \tau_{f f}^{0}$, where $\dot{M}_{*}$ denotes the total star formation rate arising from a cloud of mass $M_{c}$, size $L_{c}$ and mean density $\rho_{0}$.

\subsection{The Krumholz and McKee theory}

According to various simulations of hydrodynamic or MHD supersonic turbulence, the density PDF is well represented in both cases by a lognormal form,

$$
\begin{aligned}
\mathcal{P}(\delta) & =\frac{1}{\sqrt{2 \pi \sigma_{0}^{2}}} \exp \left(-\frac{(\delta-\bar{\delta})^{2}}{2 \sigma_{0}^{2}}\right), \delta=\ln \left(\rho / \rho_{0}\right) \\
\bar{\delta} & =-\sigma_{0}^{2} / 2, \sigma_{0}^{2}=\ln \left(1+b^{2} \mathcal{M}^{2}\right),
\end{aligned}
$$

where $\mathcal{M}$ is the Mach number and $b \simeq 0.5$ (Federrath et al. 2010).

The essence of the KM analysis is to assume that there is a critical density, $\rho_{\text {crit }}$, above which star formation is 
occuring. Then, the SFR (eqn.(20) of KM) is simply obtained by estimating the fraction of gas with density larger than $\rho_{\text {crit }}$,

$$
S F R_{f f}=\epsilon \frac{\tau_{f f}^{0}}{\tau_{f f, c r} \phi_{t}} \int_{\ln \widetilde{\rho}_{c r i t}}^{\infty} \widetilde{\rho} \mathcal{P}(\delta) d \widetilde{\delta},
$$

with $\widetilde{\rho}=\rho / \rho_{0}$. KM further assume that $\tau_{f f, c r} \simeq \tau_{f f}^{0}$.

In this expression, $\epsilon$ is the (supposedly massindependent) efficiency with which the mass within the collapsing prestellar cores is converted into stars. Calculations (e.g. Matzner \& McKee 2000, Ciardi \& Hennebelle 2010) as well as observations (e.g. André et al. 2010 ) suggest that $\epsilon \simeq 0.3-0.5$. The parameter $\phi_{t}$ corresponds to the time needed for a self-gravitating fluctuation to be replenished. KM estimate it to be of the order of a few, in agreement with the analysis we propose in the appendix.

In KM, $\rho_{\text {crit }}$ is determined from the condition that the corresponding Jeans length must be equal to the sonic length. Their underlying assumption is that turbulent support will be too efficient to enable star formation at scales larger than the sonic length. This yields $\widetilde{\rho}_{\text {crit }, K M}=\left(\phi_{x} \lambda_{J 0} / \lambda_{s}\right)^{2}$, where $\phi_{x}$ is a coefficient of order unity, $\lambda_{J 0}$ is the Jeans length at the mean cloud density and $\lambda_{s}$ is the sonic length.

\subsection{The Padoan and Nordlund theory}

The expression obtained by $\mathrm{PN}$ is similar to the KM one, stated by eqn. (2), except that they do not assume $\tau_{f f}^{0}=\tau_{f f, c r}$, but instead $\tau_{f f, c r} / \tau_{f f}^{0}=\sqrt{\tilde{\rho}_{\text {crit }}}$, as indeed comes out from the integral in eqn. (2). They consider that both $\epsilon$ and $\phi_{t}$ are equal to 1, except in the magnetized case where they argue that $\epsilon \simeq 0.5$ (which appears to be the main reason for the reduced SFR in the magnetized case). With eqns. (11) and (2), this yields (eqn.(30) of $\mathrm{PN}$ )

$$
S F R_{f f}=\frac{\epsilon}{2 \phi_{t}} \widetilde{\rho}_{\text {crit }}^{1 / 2}\left[1+\operatorname{erf}\left(\frac{\sigma_{0}^{2}-2 \ln \left(\widetilde{\rho}_{\text {crit }}\right)}{2^{3 / 2} \sigma_{0}}\right)\right] .
$$

The main difference with the KM model, however, resides in the choice of $\rho_{\text {crit }}$. In PN, this latter is obtained by requiring that the corresponding Jeans length be equal to the typical thickness of the shocked layer, inferred by combining isothermal shock jump conditions and a turbulent velocity scaling $v \propto l^{0.5}$. This yields $\widetilde{\rho}_{\text {crit }, P N} \simeq 0.067 \theta^{-2} \alpha_{\text {vir }} \mathcal{M}^{2}$, where $\theta \approx 0.35$ is the ratio of the cloud size over the turbulent integral scale and $\alpha_{v i r}$ is the virial parameter, $\alpha_{v i r}=2 E_{\text {kin }} / E_{\text {grav }}=$ $5 V_{0}^{2} /\left(\pi G \rho_{0} L_{c}^{2}\right)$, where $V_{0}$ is the rms velocity within the cloud, representative of the level of turbulent vs gravitational energy in the cloud (eqns.(8-9) of PN).

\subsection{The Hennebelle and Chabrier theory}

In the $\mathrm{HC}$ theory of star formation (see $\mathrm{HC} 08, \mathrm{HC} 09$ ) prestellar cores are the outcome of initial density fluctuations that isolate themselves from the surrounding medium under the action of gravity. These fluctuations are determined by identifying in the cloud's random field of density fluctuations the structures of mass $M$ which at scale $R$ are gravitationally unstable, according to the virial theorem. This condition defines a scale-dependent (log)-density threshold, $\delta_{R}^{c}=\ln \left(\rho_{c}(R) / \rho_{0}\right)$, or equivalently a scale-dependent Jeans mass, $M_{R}^{c}$

$$
M_{R}^{c}=a_{J}^{2 / 3}\left(\frac{\left(C_{s}\right)^{2}}{G} R+\frac{V_{0}^{2}}{3 G}\left(\frac{R}{1 \mathrm{pc}}\right)^{2 \eta} R\right),
$$

where $C_{s}$ is the sound speed, $\mathrm{G}$ the gravitational constant, $a_{J}$ a constant of order unity while $V_{0}$ and $\eta \simeq 0.4$ determine the rms velocity:

$$
\left\langle V_{\mathrm{rms}}^{2}\right\rangle=V_{0}^{2} \times\left(\frac{R}{1 \mathrm{pc}}\right)^{2 \eta} .
$$

A fluctuation of scale $R$ will be replenished within a typical crossing time $\tau_{R}$, and will thus be replenished a number of time equal to $\tau_{f f}^{0} /\left(\phi_{t} \tau_{R, f f}\right)$, where $\tau_{R, f f}=\tau_{R} / \phi_{t}$ is the freefall time at scale $R$ (see appendix), i.e. at density $\rho_{R} \sim M_{R} / R^{3}$. Including this condition into the $\mathrm{HC}$ formalism yields, after some algebra, for the numberdensity mass spectrum of gravitationally bound structures, $\mathcal{N}(M)=d(N / V) / d M$ :

$$
\mathcal{N}\left(M_{R}\right) \simeq \frac{\rho_{0}}{M_{R}} \frac{d R}{d M_{R}} \times\left(-\frac{d \delta_{R}}{d R} e^{\delta_{R}}\left(\frac{\tau_{f f}^{0}}{\tau_{R}}\right) \mathcal{P}\left(\delta_{R}\right)\right)
$$

Apart for the time ratio $\frac{\tau_{f f}^{0}}{\tau_{R}}$, this equation is similar to equation (33) of HC08 and equation (27) of HC09. According to this definition, $S F R_{f f}$ is thus given by the integral of the mass spectrum specified by equation (6):

$$
S F R_{f f}=-\frac{\epsilon}{\phi_{t}} \int_{0}^{M_{c u t}} \frac{d M}{M} \frac{d R}{d M} \frac{d \delta_{R}}{d R} \frac{\tau_{f f}^{0}}{\tau_{R, f f}} e^{\delta_{R}} \mathcal{P}\left(\delta_{R}\right) .(7)
$$

According to eqn. (4), $M_{\text {cut }}$ correponds to the mass associated with the largest size fluctuations that can turn unstable in the cloud, $y_{c u t}=2 R / L_{c}$. We verified that, as long as $y_{c u t}$ is not too small, the results depend only weakly on its value (Hennebelle \& Chabrier, in prep.). In the following, we will pick $y_{c u t} \approx 0.1$ as our fiducial value.

A few remarks are worth discussing at this stage. First, in the present theory there is no explicitly introduced critical scale or density for star formation, as we sum up over all gravitationally unstable cores, irrespectively of their scale or density. This is achieved through the multiscale analysis expressed by eqns. (6)-(7). Indeed, turbulence is by essence a multi-scale phenomenon and introducing a critical scale does not appear clearly justified. Indeed, a piece of fluid, even if dominated by turbulence, can still collapse if it is self-gravitating.

Another essential difference with the KM and PN theories is that these latter rely on a unique characteristic collapsing time (the mean cloud freefall time in KM and the critical density freefall time in $\mathrm{PN}$ ). This can be seen from eqns. (2) and (3), where the term $\widetilde{\rho}^{1 / 2}$, taken at $\widetilde{\rho}_{\text {crit }}$, lies outside the integral. In constrast, in our theory (see eqn. (7)), the freefall density dependence of each collapsing structure is properly accounted for, as the freefall time consistently varies with mass $M$ and scale $R, \tau_{R, f f} \propto \rho_{R}^{-1 / 2}$. 


\subsection{A simplified multi-freefall theory}

Even though we stress that the SFR cannot be properly determined by a simple integral of the density PDF, because such an integral, unlike eqn. (7), does not take into account the spatial distribution of the gas, we suggest the following simplified but more consistent expression, which retains the collapsing time density-dependence, instead of eqns. (2) and (3):

$$
\begin{aligned}
S F R_{f f}^{\text {simp }} & =\epsilon \int_{\delta_{\text {crit }}}^{\infty} \frac{\tau_{f f}^{0}}{\tau_{f f}(\rho) \phi_{t}} \widetilde{\rho} \mathcal{P}(\delta) d \delta=\frac{\epsilon}{\phi_{t}} \int_{\delta_{\text {crit }}}^{\infty} \widetilde{\rho}^{3 / 2} \mathcal{P}(\delta) d \delta \\
& =\frac{\epsilon}{2 \phi_{t}} \exp \left(3 \sigma_{0}^{2} / 8\right)\left[1+\operatorname{erf}\left(\frac{\sigma_{0}^{2}-\ln \left(\widetilde{\rho}_{\text {crit }}\right)}{2^{1 / 2} \sigma_{0}}\right)\right] .(8)
\end{aligned}
$$

This SFR is larger than the ones given by eqns. (2) and (3), as shown in the next section.

We consider different choices for $\rho_{\text {crit }}$. When using $\rho_{\text {crit }, K M}$ or $\rho_{\text {crit }, P N}$, we refer to the corresponding SFR as "multi-freefall KM or PN", respectively. We also consider another value for $\rho_{\text {crit }}$, obtained by simply requiring that the Jeans length at this density is equal to $y_{c u t} L_{c}$. This corresponds to the assumption that only fluctuations smaller than a given cloud size fraction can collapse. We simply refer to this model as "multi-freefall." It is easy to check that eqn.(8) depends only weakly on $y_{\text {cut }}$, except when $y_{\text {cut }} \rightarrow 0$.

\section{RESULTS}

We now compare the $S F R_{f f}$ predicted by the various theories and confront the results to recent observations.

\subsection{Comparison between the various theories}

As in $\mathrm{KM}$ and $\mathrm{PN}$, we define the cloud properties by $\alpha_{v i r}$ and $\mathcal{M}$. Figure 1 displays $S F R_{f f}^{0}$, which corresponds to $S F R_{f f}$ for $\epsilon=1$ and $\phi_{t}=1$, obtained with different formalisms, for various values of the virial parameter, and for three typical Mach numbers, namely $\mathcal{M}=16,9$ and 4 .

Both the $\mathrm{HC}$ and the multi-freefall models are larger by a factor $\sim 2-3$ than PN and by at least an order of magnitude than KM. This stems from the fact that, when taking into account the density-dependence of the structure collapsing times, (i) dense regions collapse fast, and (ii) fluctuations denser than $\rho_{\text {crit }}$ have a smaller free-fall time than the ones at $\rho_{\text {crit }}$, globally increasing the value of $S F R_{f f}^{0}$. When such a density-dependence is properly accounted for, all SFR determinations are in better agreement. Nevertheless, some differences persist between the various multi-freefall models. This stems from the choice of $\rho_{\text {crit }}$. Indeed, the choice of $\widetilde{\rho}_{\text {crit }}$ in the KM and PN theories (see $\S 2.1$ and 2.2 ) yields $y_{c u t} \approx \mathcal{M}^{-2}$ and thus corresponds to very small values of $y_{c u t}$, implying that only small Jeans masses (or conversely only very dense structures) are taken into account in these models.

Two interesting trends can be inferred from Fig. 1. First, increasing the virial parameter leads to a decrease of the SFR, with a severe reduction above some typical value of $\alpha_{v i r}$, which decreases with decreasing Mach number. This naturally arises from the fact that, as $\alpha_{v i r}$ increases, the increasing contribution of kinetic energy over potential energy prevents gravitational collapse and thus inhibits star formation, a point already noticed by
KM and PN. Second, the SFR increases, although modestly, with the Mach number. This is because increasing the Mach number extends the core mass function (CMF) into the low-mass domain (see PN and $\mathrm{HC} 08$ ) and, as small-scale structures have shorter free-fall times, this increases the number of collapsing small cores, thus the SFR. This positive dependence of the SFR upon $\mathcal{M}$ is in agreement with the results of PN but contrasts with the ones of KM (see their equation 30), as seen in the figure. Such a decreasing dependence of the SFR with increasing Mach in the KM theory clearly stems from the fact that the $\widetilde{\rho}_{\text {crit }}^{1 / 2}$ term (see eqn.(3)) is lacking in their eqn. (20).

\subsection{Comparison with observations.}

Star forming giant molecular clouds in the Milky Way have masses $10^{3} \lesssim M_{c} / M_{\odot} \lesssim 3 \times 10^{6}$, with $\mathcal{M} \approx 4-30$ and $\alpha_{v i r} \approx 0.3-3$. The observed SFR per cloud free-fall time lies in the range $0.03 \lesssim S F R_{f f} \lesssim 0.3$, with a mean value $\left\langle S F R_{f f}\right\rangle \approx 0.16$ (Murray 2011, although see Feldmann \& Gnedin 2011 for caution). Evans et al. (2009) and Heiderman et al. (2010) find SFR's in the range $\approx 0.02-0.12$ for nearby molecular clouds and $\approx 0.03-0.5$ for massive star-forming dense clumps, yielding a mean value $\approx 0.1$, about an order of magnitude larger than the values predicted by KM. Krumholz \& Tan (2007, Fig. 5) report lower values at low density. At high density $\left(\gtrsim 10^{4}\right.$ $\mathrm{cm}^{-3}$ ), however, two of their three data (ONC and CS(54)), are compatible with the aforementioned mean value:1. According to our calculations (see Fig. 1), for such cloud/clump characteristics, $S F R_{f f}^{0}$ is predicted to lie within the range $\approx 0.3-3$. As discussed in $\S 2.1$ and in the appendix, the effective SFR is $S F R_{f f}=(\epsilon / \phi) \times S F R_{f f}^{0}$, where $\epsilon / \phi_{t} \approx 0.1-0.2$. Therefore, according to the present calculations, theories based on a multi-freefall formalism yield SFR's per free-fall time in typical molecular clumps in the range $S F R_{f f} \approx 0.03-0.6$, going from lowdense clouds to the densest clumps, consistent with the observed values.

Figure 2 displays the SFR per unit area, $\dot{\Sigma}_{\star}=$ $S F R_{f f} \times \Sigma_{g} / \tau_{f f}^{0}$, with $\Sigma_{g}=L_{c} \rho_{0}=M_{c} / \pi L_{c}^{2}$, as a function of cloud surface densities, $\Sigma_{g}$, for four typical cloud sizes, $L_{c}=1,410$ and $40 \mathrm{pc}$. The clouds are assumed to follow Larson's (1981) relations and thus have velocities given by eqn. (50) and densities $n_{0} \times\left(L_{c} / 1 \mathrm{pc}\right)^{-0.7}$, where $n_{0}=10^{2}$ to $10^{4} \mathrm{~cm}^{-3}$, yielding cloud masses $M_{c}=200$ $10^{6} M_{\odot}$. From these values, $\mathcal{M}$ and $\alpha_{v i r}$ can be consistently determined. In Fig. 2, we have taken $\epsilon / \phi_{t}=0.12$

Lada et al. (2010) and Heiderman et al. (2010) data for clouds and massive clumps are shown for comparison. Clearly, almost all theories exhibit a direct correlation between the density of star formation and the gas density and, when including the $\epsilon / \phi_{t}$ factor, reproduce well the observational results, except possibly for the densest

\footnotetext{
${ }^{1}$ It must be kept in mind that all these SFR values apply to giant molecular clouds. Values inferred for entire galaxies, including the Milky Way, are substantially lower, as they include diffuse atomic or molecular gas, overestimating the amount of gas counted as star-forming gas (e.g. Heiderman et al. 2010).

2 This applies to eqn. (3) as well, whereas the true PN relation corresponds to $\epsilon / \phi_{t}=1.0$ and 0.5 in the hydro and MHD case, respectively, and should be moved upward accordingly on the figure.
} 

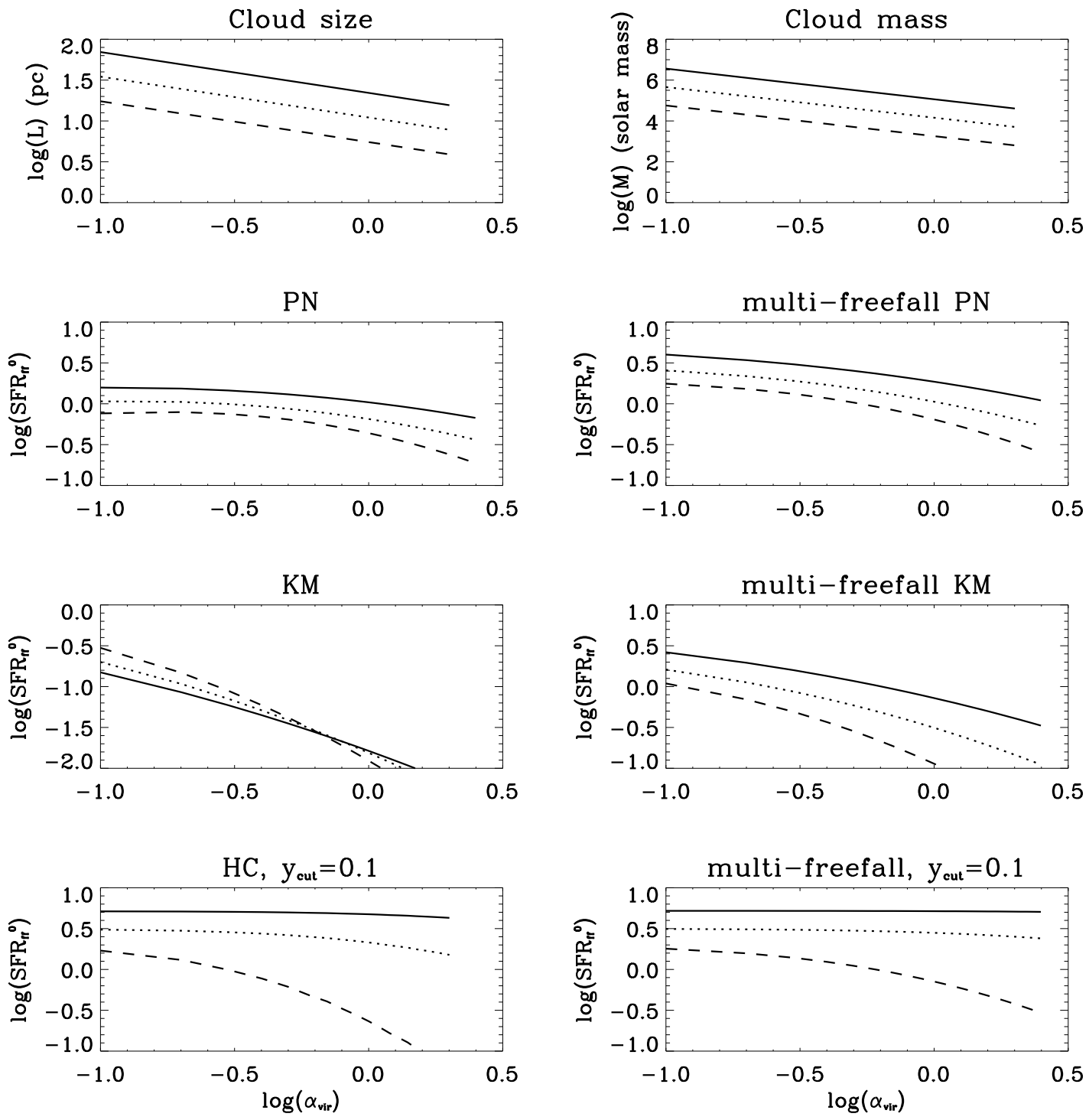

FIG. 1. $-S F R_{f f}^{0}$ as a function of $\alpha_{v i r}$ for various cloud parameters and $\mathcal{M}=16$ (solid), 9 (dot) and 4 (dash), as predicted by various theories. Top panels: cloud size and mass. Second row: Padoan \& Nordlund (2011)(left) and the corresponding "multi-freefall PN" (right). Third row: Krumholz \& McKee (2005) (left) and "multi-freefall KM" (right); Fourth row: Hennebelle \& Chabrier complete theory (eqn. (7);left) and simplified "multi-freefall" theory (eqn. (8);right) for $y_{\text {cut }}=0.1$.

clumps, where the SFR's are about a factor $\sim 5$ larger. The large spread of the data precludes a clear distinction between the theories at this stage, apart from the KM one which clearly lies well below most of the data points.

Interestingly, both the exact $\mathrm{HC}$ and "multi free-fall" calculations predict a drastic drop in the SFR below $\Sigma_{c} \approx 110-120 \mathrm{M}_{\odot} \mathrm{pc}^{-2}$ and a change of slope in the $\dot{\Sigma}_{\star} \propto \Sigma_{g}^{N}$ relation, with $N \approx 4.8$ and $N \approx 1.6$, similar to the Kennicutt-Schmidt relation $\left(N_{K S} \approx 1.4\right)$, respectively below and above $\Sigma_{c}$, in very good agreement with the observations (Heiderman et al. 2010). This density corresponds to a visual extinction $A_{V} \approx 6\left(A_{K} \approx 1\right)$. A similar density-threshold for significant dense core population has been identified in several surveys (Onishi et al. 1998, Johnstone et al. 2000, Kirk et al. 2006, Enoch et al. 2007, Lada et al. 2010, André et al. 2010). Various authors (e.g. Johnstone et al. 2004, Kirk et al. 2006, Heiderman et al. 2010) have suggested that the origin of such a density threshold is related to magnetic fields, which cannot support the gas against gravitational collapse above some density. The present calculations, however, show that there is no need to invoke magnetic field support or MHD shock conditions to get such a threshold although, as mentioned in the appendix, magnetic fields may contribute dynamically by reducing the value of $\phi_{t}$. The threshold simply stems from the fact that at the corresponding density, the size of the clumps becomes comparable to the Jeans length and thus the amount of gas appropriate to form stars drops drastically (see HC09 Fig. 8). The relative similarity between the various "multi free-fall" predictions clearly indicates that, besides the $\epsilon / \phi_{t}$ factor, the key physical quantity which determines the SFR is the density-dependence of the freefall time of the collapsing overdense regions induced by turbulence. An alternative possibility, as recently suggested by Krumholz et al. (2011) is to assume that the SFR is simply a constant factor times the cloud's or galaxy's 

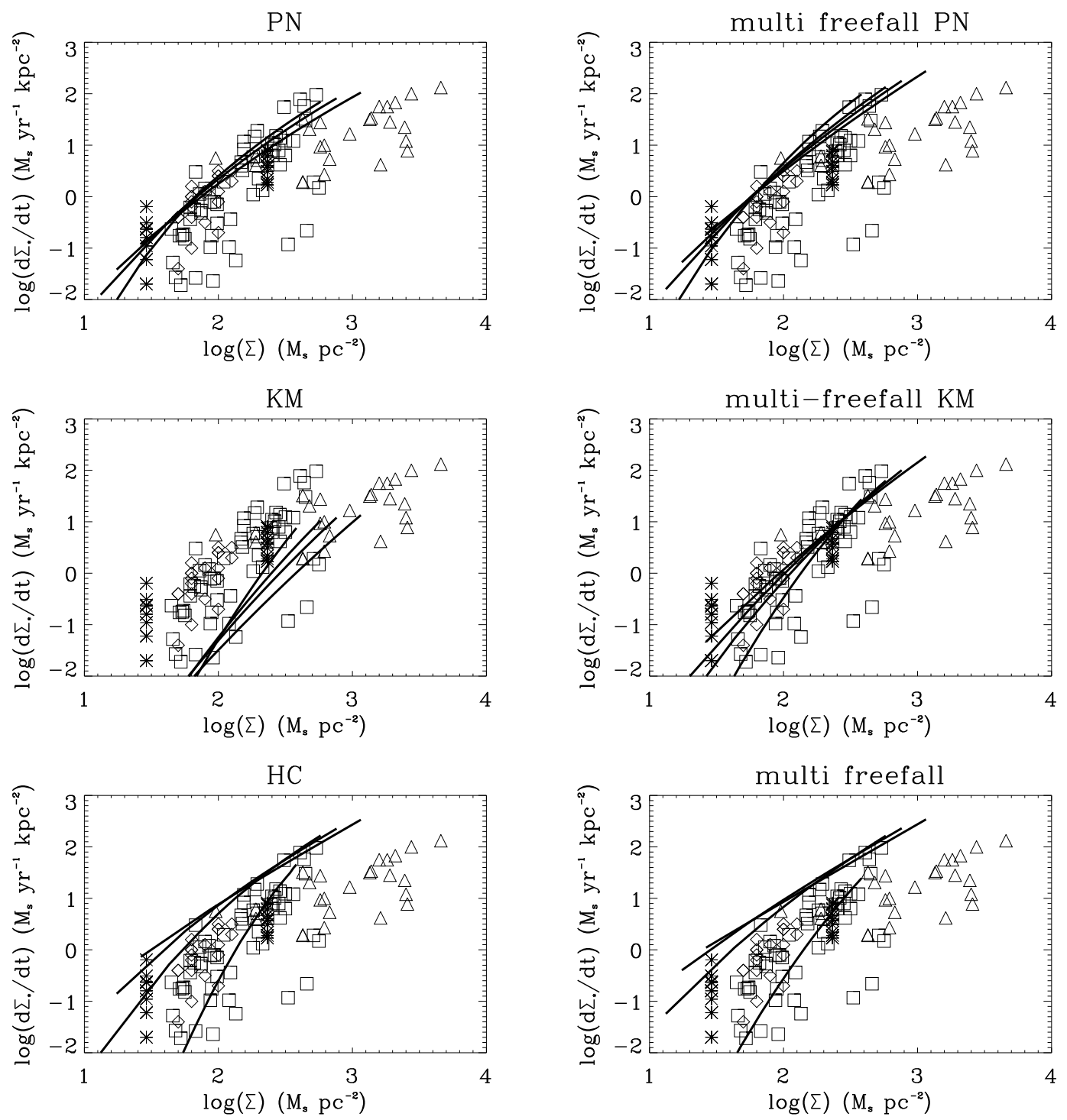

FIG. 2.- Comparison of the SFR per unit area, $\dot{\Sigma}_{\star}$, as a function of gas surface density, $\Sigma_{g}$, as predicted by the various theories illustrated in Fig. 1, with the observational determinations of Heiderman et al. (2011) for massive clumps (triangles) and molecular clouds (diamonds+squares). The stars show the data of Lada et al. (2010). The four solid lines correspond to four cloud sizes, namely $L_{c}=1,4$, 10 and $40 \mathrm{pc}$ (left to right).

volume density over mean free-fall time, although a clear physical explanation is lacking at this stage.

\section{CONCLUSION}

We have included the time dependence in our analytical theory of the IMF to determine the SFR. The theory, based on a gravoturbulent picture of star formation, yields SFR values in good agreement with various observational determinations in Galactic molecular clouds. Moreover, it naturally predicts a density threshold to get significant star formation and yields a dependence of the SFR upon gas surface density in very good agreement with the observationally inferred values, with an abrupt change of slope around the threshold. Such a threshold naturally emerges from our theory, without arbitrarily introducing a critical density.

A crucial point at the heart of the present (both complete and simplified (eq. (8))) approach is that, in contrast to previous theories, the SFR is not character- ized by a single, characteristic dynamical time in the cloud, but instead involves a density-dependent collapsing time for each turbulence-induced gravity-dominated overdense region in the cloud. Therefore, in opposite to conclusions based on previous SFR theories, the present results show that a SFR determined by turbulenceinduced density fluctuations at the early stages of star formation provides quite a consistent picture of star formation in Milky Way molecular clouds.

\section{ACKNOWLEDGEMENT}

GC acknowledges the warm hospitality of the astronomy dpt of the University of Texas, where part of this work was conducted, and A. Heiderman and N. Evans for sending their data. This research has received funding from the European Research Council under the European Community's Seventh Framework Programme (FP7/2007-2013 Grant Agreement no. 247060). 
Our estimate for the crossing time is similar to the estimate of Krumholz \& McKee (2005). The crossing time of a structure of scale $R$ is $\tau_{c t}(R)=2 R / V_{c t}$. At large scales, $V_{c t} \simeq V_{\text {rms }}$, while at small scales, below the sonic length, $V_{c t} \simeq C_{s}$. The typical time $\tau_{R}$ within which the density field is significantly modified at scale $R$, implying that a new set of fluctuations, statistically independent of the former one, has set up is $\tau_{R}=\alpha_{c t} \tau_{c t}$, with $\alpha_{c t}$ a dimensionless coefficient of the order of a few. In the Hennebelle-Chabrier theory, we select the pieces of gas which are self-gravitating. At large scales, this implies $\alpha_{g} G M / R>V_{\text {rms }}^{2}$, where $\alpha_{g}$ is a dimensionless coefficient $\left(\alpha_{g}=3 / 5\right.$ for a uniform density cloud), while a similar expression holds below the sonic length. This yields

$\tau_{R}=\frac{2 \alpha_{c t} R}{V_{\mathrm{rms}}}=2 \alpha_{c t} \sqrt{\frac{24}{\pi^{2} \alpha_{g}}} \tau_{f f}=\phi_{t} \tau_{f f}=\phi_{t} \tau_{f f}^{0} \sqrt{\rho_{0} / \rho}$,

where $\tau_{f f}=\sqrt{\frac{3 \pi}{32 G \rho}}$ is the free-fall time of a bound region of density $\rho$, and $\phi_{t} \approx 3$, yielding $\epsilon / \phi_{t} \approx 0.1-0.2$.

Note that this estimate assumes that it takes about one crossing time to rejuvenate a self-gravitating structure. However, it may happen, in particular in magnetized flows, that all perturbations do not collapse eventually (e.g. Hennebelle \& Pérault 2000), further increasing $\phi_{t}$ by a factor of a few.

\section{REFERENCES}

André, P., Men'shchikov, A., Bontemps, S. et al., 2010, A\&A, $518 \mathrm{~L}, 102$

Chabrier, G., Hennebelle, P., 2011, A\&A, arXiv 1109.2780

Ciardi, A., Hennebelle, P., 2010, MNRAS, 409L, 39

Evans, N., et al., 2009, ApJS, 181, 321

Enoch, L., Glenn, J., Evans, N. et al., 2007, ApJ, 666, 982

Federrath, C., Roman-Duval, J., Klessen, R., Schmidt, W., MacLow, M.-M., 2010, A\&A, 512, 81

Feldmann, R., Gnedin, N., 2011, ApJ, 732, 115

Heiderman, A., Evans, N., Allen, L., Huard, T., Heyer, M., 2010, ApJ, 723, 1019

Hennebelle, P., Chabrier, G., 2008, ApJ, 684, 395 (HC08)

Hennebelle, P., Chabrier, G., 2009, ApJ, 702, 1428 (HC09)

Hennebelle, P., Pérault, M., 2000, A\&A, 359, 1124

Johnstone, D., Wilson, C., Moriarty-Schieven, G., Joncas, G., Smith, G., Gregersen, E., Fich, M., 2000, ApJ, 545, 327
Kirk, H., Johnstone, D., Di Francesco, J., 2006, ApJ, 646, 1009 Krumholz, M., McKee, C., 2005, ApJ, 630, 250 (KM)

Krumholz, M., Tan, J., 2007, ApJ, 654, 304

Krumholz, M., Dekel, A., McKee, C., 2011, ApJ, arXiv 1109.4150

Lada, C., Lombardi, M., Alves, J., 2010, ApJ, 724, 687

Larson, R., 1981, MNRAS, 194, 809

MacLow, M.-M., Klessen, R., 2004, Rev. Mod. Phys., 76, 125

Matzner, C.D. \& McKee, C., 2000, ApJ, 545, 364

Murray, N., 2011, ApJ, 729, 133

Onishi, T., Mizuno, A., Kawamura, A., Ogawa, H., Fukui, Y., 1998, A\&A, 502, 296

Padoan, P., Nordlund, A., 2011, ApJ, 730, 40 (PN) 